\title{
IMPLEMENTATIONS OF CONSERVATION THEOREM AND THE EXPONENTIAL RATIONAL FUNCTION METHOD TO VISCOUS BURGERS EQUATION
}

\author{
Filiz TAŞCAN ${ }^{1, *}$, Arzu AKBULUT ${ }^{1}$, Melike KAPLAN ${ }^{1}$ \\ ${ }^{1}$ Department of Mathematics-Computer, Art-Science Faculty, Eskisehir Osmangazi University, Eskisehir, Turkey
}

\begin{abstract}
In this study, we dealt with conservation laws and exact travelling wave solutions of viscous Burgers equation. We used conservation theorem for finding conservation laws for this equation. All founded conservation laws are trivial conservation laws. Then, we found the exact solutions of viscous Burgers equation using the exponential rational function method. The results obtained confirm that the proposed method is an efficient technique for analytic treatment of a wide variety of nonlinear partial differential equations. Also the obtained results are shown graphically.
\end{abstract}

Keywords: Exact solutions, Symbolic computation, Viscous Burgers equation, Conservation laws, Lie symmetries

\section{INTRODUCTION}

It is known that all conservation laws of partial differential equations (PDEs) have not physical meanings, but they are necessary for studying the integrability and reduction of PDEs [1], [2]. There are lots of powerful methods obtaining conservation laws for PDEs for example characteristic method, variational approach, symmetry and conservation law relation, multiplier approach, direct construction method for conservation laws, partial Noether approach, Noether approach, conservation theorem [3][7]. In this study we will deal with conservation theorem. This theorem was introduced by Ibragimov. This theorem is associated with Lie symmetries, formal Lagrangian equations and adjoint equations of PDEs. Every symmetry of PDEs always provides a conservation law. These conservation laws can be trivial or non-trivial [8], [9].

Nonlinear partial differential equations (NPDEs) have common usage for describing complex phenomena in miscellaneous sciences, especially in physics and engineering. The investigation of analytical exact solutions of NPDEs plays a crucial role in the study of nonlinear physical phenomena. Because new exact solutions may provide more physical information for understanding the physical phenomena and thus lead to further applications in plasma physics, solid state physics, chemical physics, chemical kinematics, biology and so on. In recent years, a variety of powerful methods for obtaining explicit travelling and solitary wave solutions of NPDEs have been proposed. For example, Hirota bilinear transformation [10], the inverse scattering transformation [11], Bäcklund and Darboux transformation [12], the transformed rational function method [13], the tanh function method [14], the exp-function method [15], the sine-cosine method [16], the modified simple equation method [17], the extended trial equation method [18], the ansatz approach [19] and so on.

The rest of this paper is organized as follows. In Section 2, the algorithm of the conservation theorem and exponential rational function method are given. In Section 3, conservation laws and the exact travelling wave solutions of viscous Burgers equation are obtained and the results are shown graphically. 


\section{PRELIMINARY}

\subsection{Algorithm of the Conservation Theorem}

We assume that the given NPDE for $\mathrm{u}(\mathrm{x}, \mathrm{t})$ to be in the form

$$
\mathrm{F}\left(\mathrm{u}, \mathrm{u}_{\mathrm{x}}, \mathrm{u}_{\mathrm{t}}, \mathrm{u}_{\mathrm{xx}}, \mathrm{u}_{\mathrm{xt}}, \mathrm{u}_{\mathrm{tt}}, \ldots\right)=0
$$

where $F$ is a polynomial in its arguments.

Suppose that, the Lie point symmetry generators for Eq.(1) is

$$
X=\xi^{x}(x, t, u) \frac{\partial}{\partial x}+\xi^{t}(x, t, u) \frac{\partial}{\partial t}+\eta(x, t, u) \frac{\partial}{\partial u}
$$

where $\xi^{\mathrm{x}}(\mathrm{x}, \mathrm{t}, \mathrm{u}), \xi^{\mathrm{t}}(\mathrm{x}, \mathrm{t}, \mathrm{u}), \eta(\mathrm{x}, \mathrm{t}, \mathrm{u})$ are the infinitesimals and the $\mathrm{k}-\mathrm{th}$ prolongation of (2) is given by

Where

$$
X^{(k)}=X+\eta_{i}^{(1)} \frac{\partial}{\partial u_{i}^{\alpha}}+\ldots+\eta_{i_{1} i_{2} \ldots i_{k}}^{(k)} \frac{\partial}{\partial u_{i_{1} i_{2} \ldots i_{k}}}, k \geq 1
$$

$$
\begin{array}{ccc}
\eta_{i}^{(1)} & = & D_{i} \eta-\left(D_{i} \xi j\right) u_{j} \\
\eta_{i_{1} i_{2} \ldots i_{k}}^{(k)} & = & D_{i_{k}} \eta_{i_{1} i_{2} \ldots i_{k-1}}^{(k-1)}-\left(D_{i_{k}} \xi^{j}\right) u_{i_{1} i_{2} \ldots i_{k-1} j}
\end{array}
$$

here $\mathrm{i}, \mathrm{j}=1,2$ and $\mathrm{i}_{\mathrm{l}}=1$ for $\mathrm{l}=1,2, \ldots, \mathrm{k}$ and $\mathrm{D}_{\mathrm{i}}$ is the total derivative operator.

Formal Lagrangian can be obtain with

$$
\mathrm{L}=\omega \mathrm{F}
$$

where $\omega(\mathrm{x}, \mathrm{t})$ is the new adjoint variable. And then we can find adjoint equation by

$$
\mathrm{F}^{*}=\frac{\delta \mathrm{L}}{\delta \mathrm{u}} \text {. }
$$

Here $\frac{\delta}{\delta \mathrm{u}}$ is the variational derivative. If we find solution of adjoint equation (4), we can obtain finite number of conservation laws.

Theorem 1 Every Lie point, Lie-Bäcklund and nonlocal symmetry of the system of $k-$ th order differential Eq.(1) yields a conservation law. Formulae of conserved vector components are

$$
\mathrm{T}^{\mathrm{i}}=\xi^{\mathrm{i}} \mathrm{L}+\frac{\delta \mathrm{L}}{\delta u_{i}}+\sum_{\mathrm{s} \geq 1} \mathrm{D}_{\mathrm{i}_{1}} \ldots \mathrm{D}_{\mathrm{i}_{\mathrm{s}}}(\mathrm{W}) \frac{\partial \mathrm{L}}{\partial \mathrm{u}_{\mathrm{i}_{1} \mathrm{i}_{2} \ldots \mathrm{i}_{\mathrm{s}}}}
$$

with formal Lagrangian given by Eq.(3) and here $W=\eta-\xi^{j} u_{j}$ and $\xi^{i}, \eta$ are the functions. The conserved vectors obtained from Eq.(5) involves the arbitrary solutions of the adjoint equation so one obtains an infinite number of conservation laws for Eq.(1) by defining $\omega$ [20].

Theorem 2 Obtained conserved vectors from (5) are a conservation laws of Eq.(1) if

$$
\mathrm{D}_{\mathrm{i}}\left(\mathrm{T}^{\mathrm{i}}\right)=0
$$

Here $D_{i}$ is the total derivative with respect to $x$ and $t[21]$. 


\subsection{Algorithm of the Exponential Rational Function Method}

We can summarize the basic steps of the exponential rational functionmethod as follows

Consider a general nonlinear partial differential equation Eq.(1), say in two independent variables $\mathrm{x}$ and t. To find exact solutions of this equation we use the travelling wave transformation

$$
u(x, t)=u(\xi), \xi=x-c t,
$$

where $c$ is the speed of travelling wave, carries Eq.(1) to a nonlinear ordinary differential equation (ODE) as follows

$$
\mathrm{Q}\left(\mathrm{u}, \mathrm{u}^{\prime}, \mathrm{u}^{\prime \prime}, \ldots\right)=0 \text {. }
$$

Here prime denotes the derivative with respect to $\xi$. According to possibility, Eq.(8) can be integrated term by term one or more times. We seek for the solutions of Eq.(8) in the following generalized form

$$
\mathrm{u}(\xi)=\sum_{\mathrm{n}=0}^{\mathrm{m}} \frac{\mathrm{a}_{\mathrm{n}}}{\left(1+\mathrm{e}^{\xi}\right)^{\mathrm{n}}}
$$

in which $a_{n}\left(a_{m} \neq 0\right)$ are constants to be determined later.

Step 1: Firstly, we apply wave transformation (7) to Eq.(1), we obtain reduced Eq.(8).

Step 2: By balancing the highest order linear term with the highest order nonlinear term in Eq.(8), we obtain the balancing number $\mathrm{m}$.

Step 3: We substitute Eq.(9) into Eq.(8) and collect all the coefficients of $\mathrm{e}^{\mathrm{n} \xi}(\mathrm{n}=0,1, \ldots)$ to zero. Afterwards we equate each coefficient of this polynomial to zero and we find a set of algebraic equations.

Step 4: We solve this system with the aid of Maple packet program to find the values of $a_{n}$ and $c$.

Step 5: Finally, by substituting these values into Eq.(9), by use of Eq.(7), we find the exact travelling wave solutions of Eq.(1) [22].

\section{APPLICATIONS}

The viscous Burgers equation

$$
u_{t}+u_{x}-v_{x x}=0
$$

where $v$ is the viscosity coefficient. Burgers equation is a nonlinear wave propagation model, especially in fluid mechanics. This equation occurs in miscellaneous fields of applied mathematics, for instance modeling of traffic flow and gas dynamics.

\subsection{Implementation of the Theorem}

In this subsection, we will obtain conservation laws of viscous Burgers equation. Firstly, we can find formal Lagrangian for Eq.(10) in the following form

$$
\mathrm{L}=\omega\left(\mathrm{u}_{\mathrm{t}}+\mathrm{uu}_{\mathrm{x}}-\mathrm{vu}_{\mathrm{xx}}\right) .
$$

If we apply variational derivative to Eq.(10), we obtain adjoint equation with 


$$
\mathrm{F}^{*}=-\mathrm{w}_{\mathrm{x}} \mathrm{u}-\mathrm{vw}_{\mathrm{xx}}-\mathrm{w}_{\mathrm{t}}=0
$$

When we substitute $u$ instead of $\omega$ in Eq.(12), we can not obtain Eq.(10); because of this situation Eq.(10) is not self adjoint. We can say that $\omega=1$ is a solution for Eq.(12).

Eq.(10) admits following five Lie point symmetry generators are given by

$$
\mathrm{X}_{1}=\frac{\partial}{\partial \mathrm{t}}, \mathrm{X}_{2}=\frac{\partial}{\partial \mathrm{x}}, \mathrm{X}_{3}=\mathrm{t} \frac{\partial}{\partial \mathrm{x}}+\frac{\partial}{\partial \mathrm{u}}, \mathrm{X}_{4}=\mathrm{x} \frac{\partial}{\partial \mathrm{x}}+2 \mathrm{t} \frac{\partial}{\partial \mathrm{t}}-\mathrm{u} \frac{\partial}{\partial \mathrm{u}}, \mathrm{X}_{5}=\mathrm{xt} \frac{\partial}{\partial \mathrm{x}}+\mathrm{t}^{2} \frac{\partial}{\partial \mathrm{t}}+(\mathrm{x}-\mathrm{ut}) \frac{\partial}{\partial \mathrm{u}}
$$

In addition, above Lie-point symmetry generators's commutator table is

\begin{tabular}{|l|l|l|l|l|l|}
\hline$\left[\mathrm{X}_{\mathrm{i}}, \mathrm{X}_{\mathrm{j}}\right]$ & $\mathrm{X}_{1}$ & $\mathrm{X}_{2}$ & $\mathrm{X}_{3}$ & $\mathrm{X}_{4}$ & $\mathrm{X}_{5}$ \\
\hline $\mathrm{X}_{1}$ & 0 & 0 & $\mathrm{X}_{2}$ & $2 \mathrm{X}_{1}$ & $\mathrm{X}_{4}$ \\
\hline $\mathrm{X}_{2}$ & 0 & 0 & 0 & $\mathrm{X}_{2}$ & $\mathrm{X}_{3}$ \\
\hline $\mathrm{X}_{3}$ & $-\mathrm{X}_{2}$ & 0 & 0 & $-\mathrm{X}_{3}$ & 0 \\
\hline $\mathrm{X}_{4}$ & $-2 \mathrm{X}_{1}$ & $-\mathrm{X}_{2}$ & $\mathrm{X}_{3}$ & 0 & $2 \mathrm{X}_{5}$ \\
\hline $\mathrm{X}_{5}$ & $-\mathrm{X}_{4}$ & $-\mathrm{X}_{3}$ & 0 & $-2 \mathrm{X}_{5}$ & 0 \\
\hline
\end{tabular}

Now, we will obtain conservation laws of given equation for all founded Lie point symmetry generators. We can expand formula (5) for Eq.(10) with

$$
\begin{gathered}
\mathrm{T}^{\mathrm{x}}=\xi^{\mathrm{x}} \mathrm{L}+\mathrm{W}\left[\frac{\partial \mathrm{L}}{\partial \mathrm{u}_{\mathrm{x}}}-\mathrm{D}_{\mathrm{x}}\left(\frac{\partial \mathrm{L}}{\partial \mathrm{u}_{\mathrm{xx}}}\right)\right]+\mathrm{D}_{\mathrm{x}}(\mathrm{W})\left[\frac{\partial \mathrm{L}}{\partial \mathrm{u}_{\mathrm{xx}}}\right], \\
\mathrm{T}^{\mathrm{t}}=\xi^{\mathrm{t}} \mathrm{L}+\mathrm{W}\left[\frac{\partial \mathrm{L}}{\partial \mathrm{u}_{\mathrm{t}}}\right]
\end{gathered}
$$

\section{Case 1:}

When $\omega=1$ and $X_{1}=\frac{\partial}{\partial t}$, we get

and then we obtain following conserved vectors

$$
\mathrm{W}=-\mathrm{u}_{\mathrm{t}}
$$

$$
\begin{gathered}
\mathrm{T}_{1}^{\mathrm{x}}=-\mathrm{u}_{\mathrm{t}} \mathrm{u}+\mathrm{vu}_{\mathrm{xt}}, \\
\mathrm{T}_{1}^{\mathrm{t}}=\mathrm{uu}_{\mathrm{x}}-\mathrm{vu}_{\mathrm{xx}} .
\end{gathered}
$$

Conserved vectors (14) are satisfy divergence condition, so they are trivial conservation laws.

\section{Case 2 :}

If we consider $\mathrm{X}_{2}=\frac{\partial}{\partial \mathrm{x}}$, we obtain $\mathrm{W}=-\mathrm{u}_{\mathrm{x}}$. Substituting (11), $\omega=1$ and $\mathrm{W}=-\mathrm{u}_{\mathrm{x}}$ in (13), conserved vectors are found with

$$
\begin{gathered}
\mathrm{T}_{2}^{\mathrm{x}}=\mathrm{u}_{\mathrm{t}} \\
\mathrm{T}_{2}^{\mathrm{t}}=-\mathrm{u}_{\mathrm{x}} .
\end{gathered}
$$

Conserved vectors (15) satisfy (6), so they are trivial conservation laws for Eq.(10).

\section{Case 3:}

When $\mathrm{X}_{3}=\mathrm{t} \frac{\partial}{\partial \mathrm{x}}+\frac{\partial}{\partial \mathrm{u}}$, we find following conserved vectors

$$
\begin{aligned}
& \mathrm{T}_{3}^{\mathrm{x}}=\mathrm{tu}_{\mathrm{t}}+\mathrm{u}, \\
& \mathrm{T}_{3}^{\mathrm{t}}=1-\mathrm{tu}_{\mathrm{x}} .
\end{aligned}
$$

If we apply divergence condition to (16), we obtain 


$$
\mathrm{D}_{\mathrm{x}}\left(\mathrm{T}_{3}^{\mathrm{x}}\right)+\mathrm{D}_{\mathrm{t}}\left(\mathrm{T}_{3}^{\mathrm{t}}\right)=\mathrm{tu}_{\mathrm{xt}}+\mathrm{u}_{\mathrm{x}}-\mathrm{tu}_{\mathrm{xt}}-\mathrm{u}_{\mathrm{x}}
$$

so they are trivial conservation laws.

0 ,

\section{Case 4:}

Now, let us find conservation laws corresponds to the operator $\mathrm{X}_{4}=\mathrm{x} \frac{\partial}{\partial \mathrm{x}}+2 \mathrm{t} \frac{\partial}{\partial \mathrm{t}}-\mathrm{u} \frac{\partial}{\partial \mathrm{u}}$. For this operator, the infinitesimals are $\xi^{\mathrm{x}}=\mathrm{x}, \xi^{\mathrm{t}}=2 \mathrm{t}, \eta=-\mathrm{u}$ and we get

$\mathrm{W}=-\mathrm{u}-\mathrm{xu}_{\mathrm{x}}-2 \mathrm{tu}_{\mathrm{t}}$ and the corresponding conserved vector of Eq.(10) as

$$
\begin{gathered}
T_{4}^{\mathrm{x}}=\mathrm{xu}_{\mathrm{t}}-\mathrm{u}^{2}-2 \mathrm{utu}_{\mathrm{t}}+2 \mathrm{vu}_{\mathrm{x}}+2 \mathrm{vtu}_{\mathrm{xt}}, \\
\mathrm{T}_{4}^{\mathrm{t}}=2 \mathrm{tuu}_{\mathrm{x}}-2 \mathrm{tvu}_{\mathrm{xx}}-\mathrm{u}-\mathrm{xu}_{\mathrm{x}} .
\end{gathered}
$$

here we used $\omega=1$. The divergence condition $\mathrm{D}_{\mathrm{x}}\left(\mathrm{T}_{4}^{\mathrm{x}}\right)+\mathrm{D}_{\mathrm{t}}\left(\mathrm{T}_{4}^{\mathrm{t}}\right)$ is equal to zero, so conserved vectors (17) are trivial.

\section{Case 5:}

Similarly, for operator $X_{5}=x t \frac{\partial}{\partial x}+t^{2} \frac{\partial}{\partial t}+(x-u t) \frac{\partial}{\partial u}$, when $\omega=1$, corresponding conserved vector of Eq.(10) as

$$
\begin{gathered}
T_{5}^{x}=x t u_{t}+u x-u^{2} t-u t^{2} u_{t}-v+2 v u_{x} t+v t^{2} u_{x t}, \\
T_{5}^{t}=t^{2} u u_{x}-t^{2} v u_{x x}+x-u t-t x u_{x} .
\end{gathered}
$$

The divergence condition (6) is zero, so we can say that founded conserved vectors (18) are trivial conservation laws.

\subsection{Implementatıon of the Exponential Rational Function Method}

According the exponential rational function method, introducing the traveling wave transformation Eq.(7), Eq.(10) is transformed to the following ODE

Integrating Eq.(19) with respect to $\xi$, we obtain

$$
-\mathrm{cu}^{\prime}+\mathrm{uu}^{\prime}-\mathrm{vu} \mathrm{u}^{\prime \prime}=0
$$

$$
-\mathrm{cu}+\frac{\mathrm{u}^{2}}{2}-\mathrm{vu}^{\prime}=0
$$

Here the integration constant is taken as zero. Balancing the highest order derivative term $\mathrm{u}^{\prime}$ with the highest order nonlinear term $\mathrm{u}^{2}$ gives $\mathrm{m}=1$. Then the solution turns into

$$
\mathrm{u}(\xi)=\mathrm{a}_{0}+\mathrm{a}_{1} \mathrm{e}^{\xi},
$$

from Eq.(9). Substituting Eq.(21) into Eq.(20) and collecting the coefficients of $\mathrm{e}^{2 \xi}, \mathrm{e}^{2 \xi}, \mathrm{e}^{2 \xi}$ to zero, we get a set of algebraic equations

$$
\begin{array}{ccc}
\mathrm{e}^{2 \xi} & : & -c \mathrm{a}_{0}+\frac{\mathrm{a}_{0}^{2}}{2}=0, \\
\mathrm{e}^{\xi} & : & -2 \mathrm{ca}_{0}+\mathrm{a}_{0}^{2}+\mathrm{va}_{1}-\mathrm{ca}_{1}+\mathrm{a}_{0} \mathrm{a}_{1}=0, \\
\mathrm{e}^{0 \xi} & : & -\mathrm{ca}_{0}+\frac{\mathrm{a}_{0}^{2}}{2}+\frac{\mathrm{a}_{1}^{2}}{2}-\mathrm{ca}_{1}+\mathrm{a}_{0} \mathrm{a}_{1}=0 .
\end{array}
$$


From the solutions of the system above with the aid of Maple, we have two different cases. We can present these cases as follows.

\section{Case 1:}

$$
\mathrm{a}_{0}=0, \mathrm{a}_{1}=2 \mathrm{v}, \mathrm{c}=\mathrm{v},
$$

By substituting the values Eq.(22) into the exact solution Eq.(21), by use of Eq.(7), we obtain the exact travelling wave solutions of viscous Burgers equation as follows:

$$
u(x, t)=\frac{2 v}{1+\cosh (x-v t)+\sinh (x-v t)}
$$

When the parameters are given special values, the solitary waves are derived from the travelling waves. We plot this solution of the viscous Burgers equation when $\mathrm{v}=1$ within the interval $0 \leq \mathrm{x}, \mathrm{t} \leq 10$.

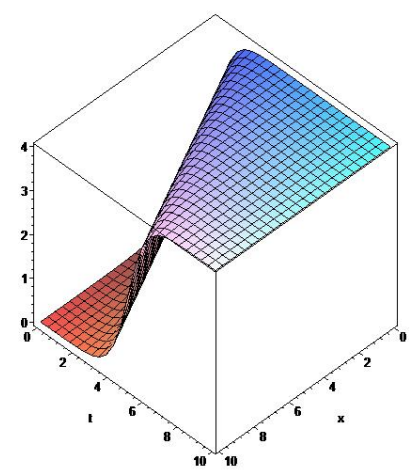

Figure 1. Graphical representation of Case 1 for viscous Burgers equation

\section{Case 2:}

$$
\mathrm{a}_{0}=-2 \mathrm{v}, \mathrm{a}_{1}=2 \mathrm{v}, \mathrm{c}=-\mathrm{v}
$$

When we substitute Eq.(23) into the exact solution Eq.(21), by use of Eq.(7), we find the exact travelling wave solutions of viscous Burgers equationa as follows:

$$
u(x, t)=-2 v\left(\frac{\cosh (x+v t)+\sinh (x+v t)}{1+\cosh (x+v t)+\sinh (x+v t)}\right)
$$

When the parameters are given special values, the solitary waves are derived from the travelling waves. We plot this solution of the viscous Burgers equation when $v=1$ within the interval $0 \leq x, t \leq 10$.

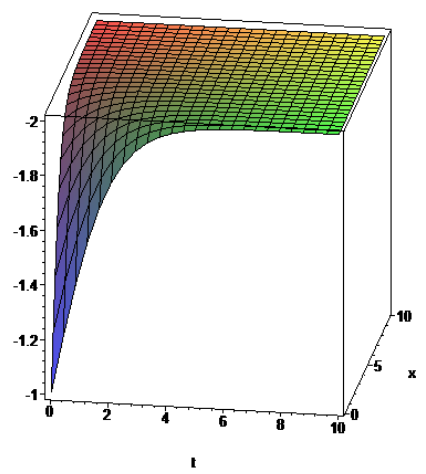

Figure 2. Graphical representation of Case 2 for viscous Burgers equation 


\section{CONCLUSION}

In this study, we considered viscous Burgers equation for finding conservation laws and exact travelling wave solutions. Firstly, we gave needed informations about conservation theorem and exponential rational function method in section 2 . Then we applied this methods to our equation in section 3 . We obtained trivial conservation laws in subsection 3.1. We gave five Lie point symmetry generators which are admitted by viscous Burgers equation and commutator table is given. Then, we obtained formal Lagrangian and adjoint equation for given equation, we said that $\omega=1$ is a solution of adjoint equation. We obtained five trivial conservation laws for viscous Burgers equation different from each other. These founded conservation laws are trivial because of divergence condition is satisfied.

In subsection 3.2, the exponential rational function method has been successfully employed to solve viscous Burgers equation. The obtained solutions may be worthwhile for explanation of some physical phenomena accurately. The used method has many advantages: it is straight forward and compendious. Graphical representation is an important instrument for communication and it illustrates obviously the solutions of the problems. So, we gave the graphical representation of the obtained solutions.

Finally, we can note that, when we compare the solutions obtained in this paper with the given ones in, we can remark that our solutions are new. Also since the solution procedure is simple and direct, this work going to be useful for the literature.

\section{REFERENCES}

[1] Compére G. Symmetries and conservation laws in Lagrangian gauge theories with applications to the mechanics of black holes and to Gravity in three dimensions. Ph.D. thesis, Université Libre de Bruxells Faculté des Sciences, 2007.

[2] Olver PJ. Application of Lie Groups to Differential Equations Springer-Verlag, New York, 1993.

[3] Naz R, Mahomed FM, Mason DP. Conservation laws via the partial Lagrangian and invariant solutions for radial and two-dimensional free jets Non. Analy.: Real World Appl., 10:pp. 3457-3465, 2009.

[4] Yasar E. Variational principles and conservation laws to the Burridge-Knopoff equation. Nonlinear Dynamics 2008; 54: 307-312.

[5] Wang G, Kara AH, Fakhar K. Vega-Guzmand J, Biswas A. Group analysis, exact solutions and conservation laws of a generalized fifth order KdV equation. Chaos, Solitons and Fractals, 2016; 86: 815.

[6] Ibragimov NH, Khamitova R, Avdonina ED, Galiakberova LR. Conservation laws and solutions of a quantum drift-diffusion model for semiconductors. International Journal of Non-Linear Mechanics 2015; 77: 69-73.

[7] Ibragimov NH, Kolsrud T. Lagrangian Approach to Evolution Equations: Symmetries and Conservation Laws. Nonlinear Dynamics 2004; 36: 29-40.

[8] Tascan F, Yakut A. Conservation Laws and Exact Solutions with Symmetry Reduction of Nonlinear Reaction Diffusion Equations. Int. J. of Non. Sci. and Num. Simul. 2015; 16: 191-196.

[9] Ibragimov NH. A new conservation theorem. J. Math.Anal. Appl. 2007; 333: 311-328. 
[10] Hirota R. Direct method of finding exact solutions of nonlinear evolution equations, in: R. Bullough, P. Caudrey (Eds.), Backlund Transformations, Springer, Berlin, 1980.

[11] Vakhnenko VO, Parkes EJ, Morrison AJ. A Bäcklund transformation and the inverse scattering transform method for the generalised Vakhnenko equation. Chaos, Solitons \& Fractals 2003; 17: 683692.

[12] Wadati M, Sanuki H, Konno K. Relationships among inverse method Backlund transformation and an infinite number of conservation laws. Prog. Theor. Phys. 1975; 53: 419-436.

[13] Ma WX, Lee JH. A transformed rational function method and exact solutions to the $3+1$ dimensional Jimbo-Miwa equation. Chaos, Solitons and Fractals 2009; 42: 1356-1363.

[14] Wazwaz AM. The tanh method for travelling wave solutions of nonlinear equations. Applied Mathematics and Computation 2004; 154 (3): 713-723.

[15] Bekir A, Boz A. Exact solutions for nonlinear evolution equations using exp-function method Phys. Lett. A 2008; 372: 1619-1625.

[16] Bekir A. New exact travelling wave solutions of some complex nonlinear equations. Commun Nonlinear Sci Numer Simulat 2009; 14: 1069-1077.

[17] Mirzazadeh M. Modified Simple Equation Method and its Applications to Nonlinear Partial Differential Equations. Inf. Sci. Lett. 2014; 3 (1): 1-9.

[18] Gurefe Y, Misirli E, Sonmezoglu A, Ekici M. Extended trial equation method to generalized nonlinear partial dierential equations. Applied Mathematics and Computation 2013; 219: 5253-5260.

[19] Younis M, Ali S, Mahmood SA. Solitons for compound KdV-Burgers equation with variable coefficients and power law nonlinearity. Nonlinear Dynamics 2015; 81: 1191-1196.

[20] Yakut A. Conservation Laws for Partial Differential Equations, Master Thesis, Eskisehir Osmangazi University, Eskisehir, Turkey, 2012.

[21] Akbulut A, Kaplan M, Tascan F. Conservation laws and Exact Solutions of Phi-Four (Phi-4) Equation via the (G'/G, 1/G)-Expansion Method. Z. Naturforsch 2016; 71(5)a: 439-446.

[22] Yusufoglu E, Bekir A. A travelling wave solution to the Ostrovsky equation. Applied Mathematics and Computation 2007, 186: 256-260.

[23] Islam Md H, Khan K, Akbar MA, Salam Md A. Exact traveling wave solutions of modified KdVZakharov-Kuznetsov equation and viscous Burgers equation. SpringerPlus 2014; 33: 105. 